\title{
Development of a Flash-based Schedule Management Tool for Smart Devices
}

\author{
Jang, Myunghoun* \\ Department of Architectural Engineering, Jeju National University, Jeju, 690-756, Korea
}

\begin{abstract}
Schedule management of construction work is critical to completing construction projects within a given period of time. Many efforts have been made and multiple tools have been developed to support the management of construction projects using smart devices such as smartphones and tablet PCs. This study proposed a method for managing construction schedules via a smart device using Flash. The interactive and graphic properties of Flash enable the development of an app that can allow a field manager to manage schedules easily and efficiently. A prototype was implemented using Adobe Flex Builder, and a sample network was applied in a smartphone. It is convenient for a construction manager to manage construction schedules using the tool because Flash controls graphic components easily. In further studies, network diagrams such as CPM (Critical Path Method) should be developed and applied to the app.
\end{abstract}

Keywords : schedule management, smart device, bar chart, schedule network

\section{Introduction}

\subsection{Research background and objective}

Smart devices such as smartphones and tablet PCs have been widely adopted thanks to the convenience they offer users, including their usefulness and swiftness for checking data or making notes. With this trend, the mobile office[1], which allows a worker to work anytime and anywhere using a mobile device, has also gained popularity, along with the smart work system (home-based teleworking + mobile working + smart work center).

With respect to construction sites, it is now possible to change the information handling process from the office (company level) to the

Received : May 3, 2012

Revision received : June 12, 2012

Accepted : June 13, 2012

* Corresponding author : Jang, Myunghoun

[Tel: 82-64-754-3704, E-mail: jangmh@jejunu.ac.kr]

(c)2012 The Korea Institute of Building Construction, All rights reserved. construction site without a time delay (person, construction manager level)[2], and smart devices will be utilized in decision-making for construction management, including schedule management. In the schedule management of a construction project, the schedule will often need to be checked and revised, which is mostly done on a PC. Some companies are currently adopting web-based schedule management[3], and smart devices are mainly used to check information on construction progress while at construction sites. Considering this current situation, this paper proposes a flash-based schedule management tool to utilize on smartphones at construction sites.

\subsection{Research method and scope}

The research method and process to develop a schedule management tool for smart devices are as follows:

1) The tools and previous research related with construction project management on smart devices are first researched and analyzed. 
2) The management fields in a construction project that can be easily managed on the smart device were checked, and the implementation direction was drawn.

3) A schedule management tool for smart devices was developed, and then the prototype was implemented.

4) To verify the practicability of the tool, the tool was used at actual construction sites and its applicability was verified through the advice of professionals.

This research focuses on the development of a schedule management tool for smart devices including smartphones and tablet PCs. Resources management and cost accounting are excluded from the scope of research.

\section{Schedule management for smart devices}

\subsection{Schedule management applications for smartphones}

There are a variety of schedule management applications (hereinafter 'apps') for smart devices that are mostly for personal work scheduling, and for this reason they are insufficient to apply to schedule management of a construction project. There are few apps appropriate for schedule management of a construction project with a bar-charted format.

Project Schedule, MPM, Power List, and Ganttroid are existing schedule management apps for Android-based smart devices. Project Schedule enables managers to input each activity, and shows the activity in a bar chart. Different activities can be grouped, but if the grouping function is used, it is difficult to manage the activity list, and impossible to input and retrieve one activity individually.

MPM simply displays the list of activities input by the user. It provides a bar chart function in
HTML (HyperText Markup Language), but the user cannot revise the bar chart himself/herself. Power List creates activities by the user entering the start date and end date of each activity. A user can check the bar chart with a move function, but is unable to enlarge or reduce it. Ganttroid displays the schedule the user has entered in the calendar in the smart device on the screen. It provides the information in a zoomable bar-charted format that can be scrolled through easily, but the schedule cannot be revised on the screen directly. These schedule management apps are appropriate for simple notes, such as a personal schedule, but are inappropriate for checking the relationship of works in progress, completion rate and resources.

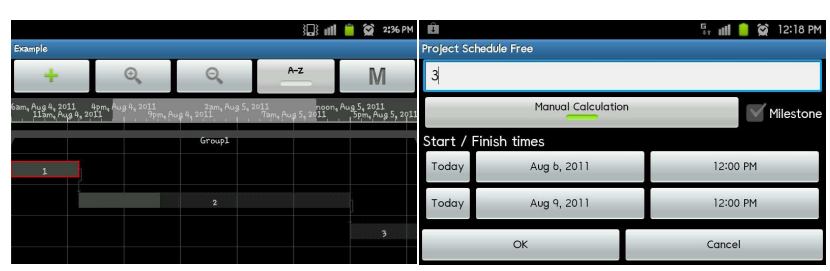

(a) Project Schedule

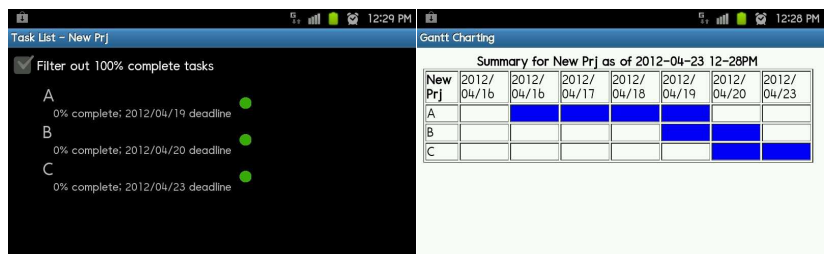

(b) MPM

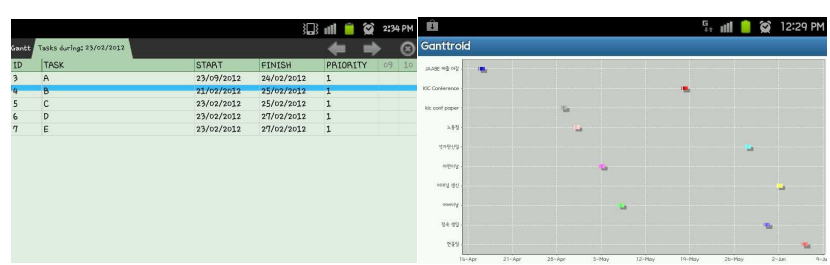

(c) Power List

(d) Ganttroid

Figure 1. Scheduling apps for Android phones

In the schedule management apps available for smartphones, it is relatively difficult to enter and edit activities due to the smartphone's small screen. In addition, it is impossible to check the entire construction schedule at a glance on the small 
screen. On a tablet PC, some apps are not supported, and images may not be displayed in an appropriate manner due to the resolution discrepancy. To resolve these problems, apps designed specifically for tablet PCs should be developed.

\subsection{Research on smart device-based construction project management}

The rapid diffusion of smart devices has led to a number of studies being carried out on the use of smartphones as a tool in construction projects. The studies considered how smartphones could be utilized in overall construction management, including safety management, material management and progress management, but few cases were concretely implemented. Most of them only presented a superficial system or development plan.

The study that researched the software types used at construction sites and analyzed the development direction of an app[4] presented an app customized for construction sites, including functions such as schedule management, communication, document management, picture/voice management, weather checking, and payment management. To resolve the information disconnect between a construction site and the office and information management structure managed at the company level, a plan to connect a smartphone with the Project Management Information System and smartphone-based system for construction site management was proposed[5]. The types of construction project management that can be carried out using a smartphone were analyzed, and the implementation scope of construction site management was presented[6]. Since the performance of a smartphone is limited, it is noted that the optimal functions should be utilized by using technologies appropriate for construction site management. While these studies are practical research on the utilization of smartphones, system components or screen design have not yet been presented concretely.

The study that proposed a PMS (Project Management Information System)-based app for smartphones for safety management of a construction project[7] presented a safety guide app by work type at the site that offered pictures, figures and text, the application concept of real-time safety management at a construction site and the user interface. However, only simple and basic content and concept were presented, including the screen composition and menu setting for site managers, and no concrete system was developed.

After bar code, QR code and RFID were considered for collecting information from mobile devices[8], a method utilizing only RFID and QR code was proposed. A materials management app [9] was presented following an analysis of the existing problems and a survey. Conventional construction management methods using IT, including methods employing PDA, bar code, RFID, web cameras, etc. are noted to have caused re-work or inefficiency due to a lack of real-time information processing technology and the separation of the management office from the construction site. The materials management app presented was designed to provide various functions, including auto-entry of information, real-time processing and checking of materials information, and location check of materials being delivered. The concept of tracking materials using the GPS function in a smartphone and entering materials using QR(Quick Response) codes was presented, but a concrete app was not developed.

There was a study that presented a method of construction progress management on a smartphone using an augmented reality app [10]. 
It provides the user interface of the smartphone app, but the system has not yet been developed. A corporation for which location information is important, such as Korea Expressway Corporation, can utilize the LBS(Location-based Service) of a smartphone. Road Management System[11] uses the LBS in managing and repairing roads, leading to reductions in time and expense. While it is appropriate for a company or corporation to utilize LBS when the road information is important, construction work is mostly done in a restricted space, and the need for LBS in construction work is relatively low.

There are some cases in which construction companies apply smartphones to their actual practice, and these include the mobile office and smart work-oriented examples. Company $G$ is planning to operate EIP(Enterprise Information Portal) + TPMS(Total Project Management System) using smartphones, and it has already applied Mobile EIP to its mail and bulletin board system. Some product improvements and cost reductions have been reported by implementing manpower management at a site and materials management based on the daily work management process through the Mobile TPMS. Mobile systems are in development and some have already been applied as exemplary cases; however, feedback to the utilization results still has a long way to go.

In the previous studies and developments, smart devices were not fully utilized. The smart devices were restrictedly used to check the information of construction sites. Furthermore, the studies that proposed system development only presented concepts, factors or ways of development, and for this reason, more active applications of smart devices should be presented.

\section{Development of a schedule management tool for smart devices}

\subsection{Development direction}

The efforts made in the previous studies are at the initial stage of using smart devices for purposes that go beyond collecting and storing data and checking the construction project's progress. For the active application of smart devices to construction management, a diversity of appropriate apps are needed, but there is a restriction on the type and number of apps that can be currently utilized. As mentioned earlier, schedule management, document management, picture/voice management, weather search, and payment management are appropriate items for customized construction site apps[4], and this study proposes a basic schedule management app for construction management.

For the schedule management of a construction project, considering that a certain level of information is required, the app should provide the function to input and revise a number of pieces of information, and the function to be linked to a site office to check and update the original and revised information at the office. Taking into account the screen size and performance of the smart devices, it is difficult to manage a whole construction project via a smart device. But the user interface should be able to be enlarged and reduced for the user's convenience when checking the detailed schedule information. In addition, a function allowing the user to adjust the schedule easily would be desirable.

\subsection{Tool development}

Apps run on smart devices are usually developed using a tool like the Java-based Eclipse. However, the prototype of the app for an Android-based 
smart device presented in this study was developed using Flash, as Flash not only provides a good graphic environment but also is user-friendly, but can also be run by diverse OS and environments including Microsoft Windows, Android and web.

Adobe Flex was used as a tool in developing the Flash app. With Flex, it is possible to develop an app that can be distributed en masse regardless of web browser type, desktop or operating system. Flex provides the function of user entering and retrieving factors in the name of control, and DataGrid was also used to show the activity name, period, start date and end date in the schedule.

\subsection{Implementation of the prototype}

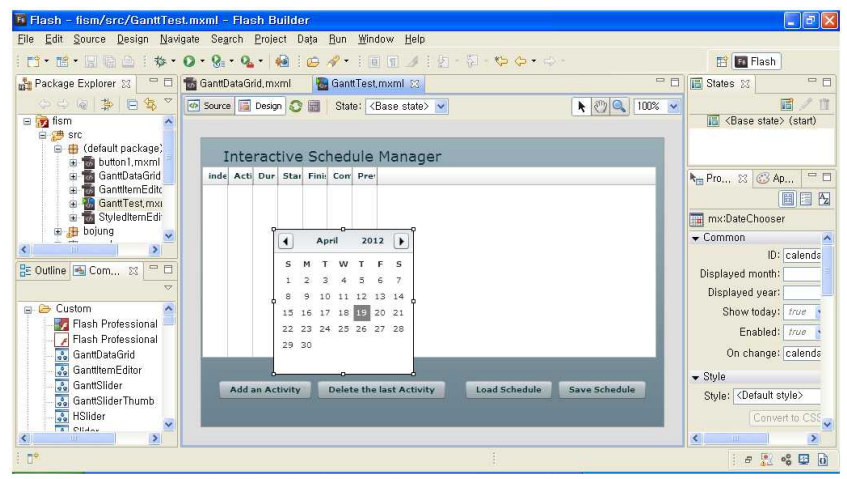

(a) Screen layout

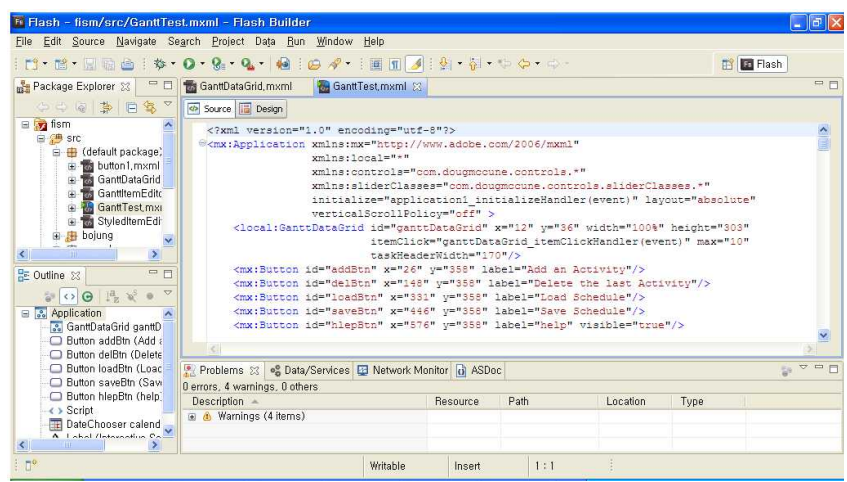

(b) Coding

Figure 2. Adobe Flash/Flex Builder (version 4.6)

To implement the prototype Flex was used, and Adobe Flash Builder was also used, particularly for the concrete implementation of the app. Figure 2 illustrates the programming using the Flash Builder. DataGrid and diverse types of buttons were placed on the screen, and the program was written in Java programming language to activate the controls on the screen. The source code written was saved in MXML (XML-based user interface markup language) format. MXML is usually used to write the screen layout for a program, and for this reason, it was used to develop the designated screen composition of and the controls to be used on the prototype app.

The prototype of the schedule management app can display an activity as shown in Figure 2(a), and the interface consists of DataGrid, activity addition/deletion button, and schedule saving and retrieving button. The calendar on which the start date and end date is entered was included in DataGrid. DataGrid was composed of Table and Graphic, each of which shows activity information and bar chart, respectively, and these were interworking on a screen. As shown in Figure 2, the detailed parts were programmed in the MXML format, including the controls to enter an activity and adjust the date.

Diverse controls are used to implement the prototype, but the code to activate the schedule management tool was written directly. The composition of the main code for prototype implementation is indicated in Figure 3. Fism. mxml is an execution function, which composes the screen of the prototype, enables a user to enter and retrieve the schedule and delivers information to other functions if necessary. FismDataGrid.mxml designates the DataGrid format, including activity name, period, start date and end date for schedule management. FismItemEditor.mxml has the function of handling the value entered or revised by the user within the DataGrid. The important variables and values used in the prototype were set in Global 
VArs.as and saved in the ActionScript format.

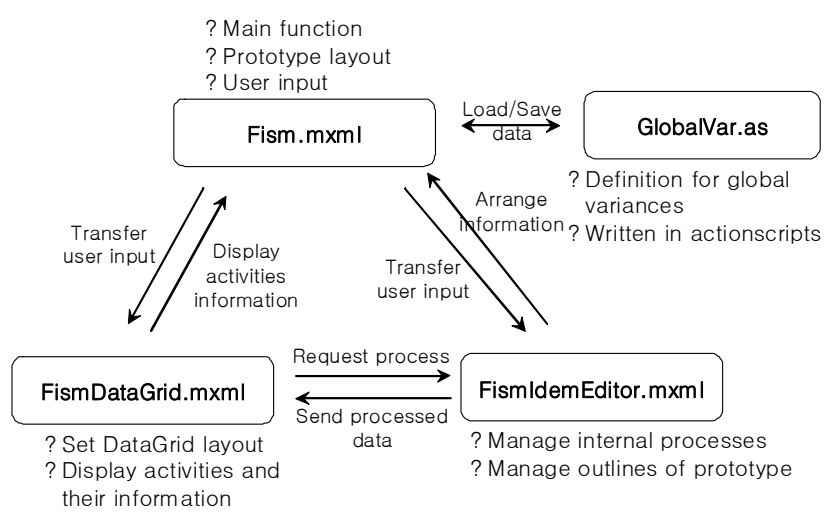

Figure 3. Internal functions of the prototype

Figure 4 is the screen of the prototype of the schedule management app developed using Flex and run on an Android-based smartphone. The prototype was shown on the screen using the SWF Player app. Run on a tablet PC using the same app, the larger screen size made it more convenient to enter and retrieve the schedule by touch. If an activity is inserted using the activity addition button on the prototype screen, a bar chart lasting for one day is created. The bar chart can be moved to the right and left by touching the bar to adjust date and period, and the completeness can also be set. When the start date or end date is selected, a calendar appears, on which the schedule can be easily adjusted even on a small smartphone screen. Users can add or delete an activity on the DataGrid screen, and the entered schedule can be saved an external file in the XML(eXtensible Markup Language) format. To evaluate the validity of the tool and prototype suggested in this study, a simple schedule was converted and applied to a smartphone and tablet PC. On the Android-based smartphone and tablet $\mathrm{PC}$, an identical screen and function were executed, and it was possible to enter and adjust a schedule on the devices.

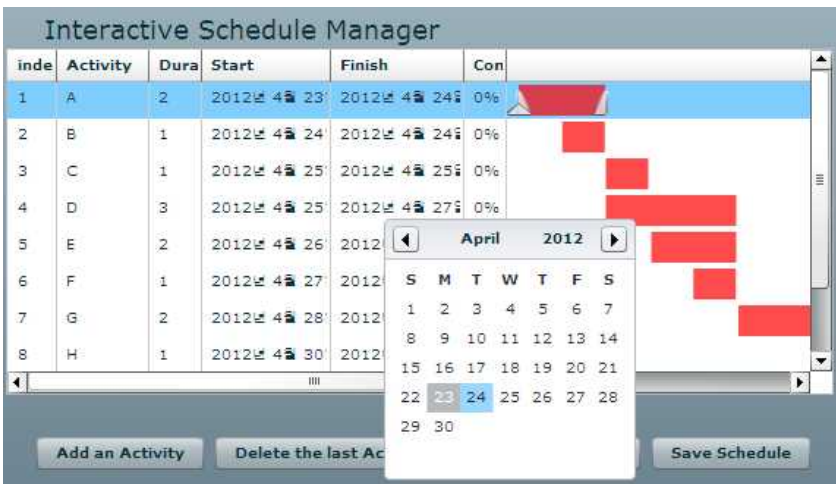

Figure 4. The prototype loaded in a smart phone based on Android OS

\subsection{Interview with professionals and analysis}

We asked some professionals to provide their advice on the practicability of the schedule management tool developed in this study. To get practical advice, the interviewees were 5 people employed by large-scale construction companies in the position of manager or higher. As shown in Table 1, we asked questions on the practicability of the web-based schedule management, necessary functions, difficulties and inconveniences found during use, and improvements.

Most of the interviewees were aware of the efforts being made to use smartphones in managing a construction project, and answered that some construction companies had already introduced or were planning to introduce such a system. In terms of the practicability of the system proposed in this study, the tool was familiar due to its Flash format, and seemed convenient for novices (beginners) to use since a simple schedule could be input with the bar chart. Some suggested that it would be better to incorporate a cost accounting function in addition to the simple schedule management function.

In terms of other opinions offered, it was noted that the app was programmed in Flash, and that it was inconvenient to enter and retrieve the activity information, and the bar chart made it difficult to 
set a period of an activity, and pick a specific start or end date as well. The enlargement and reduction of the screen size should be improved in the event of an extension or reduction in the period of an activity. As well, some asked for a function to display and revise the detailed information of the activity.

The bar chart format was appropriate, but there was no function to link and utilize the previous and next works to visualize the network. It was thought that this function should be added, in addition to a function to add the person in charge of the activity and the resources required for the activity. In addition, there were some opinions that the interface should be changed to make it more appropriate for construction project managers.

Table 1. Interviews with construction experts

\begin{tabular}{cc}
\hline Class & Contents \\
\hline Time & Sep. 2011 - Nov. 2011 \\
& Associate Manager B (HD company) \\
Associate Manager J (DS company) \\
Associate Manager K (DS company) \\
Associate Manager L (DL company) \\
Manager J (KH company) \\
Applicability to construction fields, \\
Additional necessary functions, \\
Difficulties to use, \\
and Improvements required \\
of the proposed schedule tool
\end{tabular}

\subsection{Analysis and improvements}

The development environment for a smart device is different from the environment for a general PC or the web, and this needs to be recognized in order to develop a practical app. Due to the device's small screen, the content should be easily enlarged and reduced, in order to select and revise activities. In addition, the small screen prevents various pieces of information from being displayed, and users should be able to move from one screen to another to check the information. For this reason, the design must allow users to move between screens and check the data easily. To save or retrieve schedule information from the inside of a smart device, the XML format was used, but to manage the data in a more effective manner, the Android internal database needs to be considered. For effective schedule management, the detailed information of the activity should be presented and revised, and this is an area for future work.

\section{Conclusion}

With the use of smart devices on the rise, many efforts are being made to utilize smart devices in the management of construction projects. A Flash tool for schedule management on a smart device is proposed in this research. The prototype was implemented in which with the direct control on a smart device, a data or period of an activity can be entered and revised, and the applicability of the tool to actual schedule management in a construction project was verified through interviews with professionals. In addition, difficulties and inconveniences with the tool were also found, including a lack of detailed information regarding activities, and inconveniences in selecting or setting the related information of an activity.

The Flash-based schedule management app can be applied to diverse types of OS; however, to develop an app that is optimal for smart devices, the use of tools exclusive for each OS should be considered. Furthermore, such an app should be improved to include or interwork with resources management and cost accounting, considering the improved capabilities of many smart devices. In addition, the CPM(Critical Path Method) method should be included in the schedule management tool to perform complicated schedule computation 
in a subsequent study.

\section{Acknowledgement}

This research was supported by Basic Science Research Program through the National Research Foundation of Korea(NRF) funded by the Ministry of Education, Science and Technology. Grant No. 2010-0011139.

\section{References}

1. Na SW, Lee YH, Jee SS. Security Issues and Strategies of Smart Phone and Mobile Office. CIO(Chief Information Officer) Report. 2010 Oct;26(1):2-28.

2. Youn SW, Chin SY, Park MS, Kwak MS, Choi CH. A Development of Project Management Information System using SMART Phone. Proceedings of Korea Institute of Construction Engineering and Management Annual Conference; 2010 Nov 5-6; Incheon, Korea. Seoul (Korea): Korea Institute of Construction Engineering and Management; c2010. p. 19-20.

3. Jang MH. Development of a web-based Schedule Tool Using Flash. Journal of the Korea Institute of Building Construction. 2010 Apr;10(2):51-7.

4. Ahn CS, Lee MK, Kim YJ, Kim SA, Yoon SW, Chin SY. A study on the Smart phone Application Development and Its direction Considering Construction Site Characteristics. Proceedings of Korea Institute of Construction Engineering and Management Annual Conference; 2010 Nov 5-6; Incheon, Korea. Seoul (Korea), Korea Institute of Construction Engineering and Management; c2010. p. 113-4.

5. Yoon SW, Chin SY, Park MS, Kwag MS, Choi CH. A Development of Project Management Information System using SMART Phone. Proceedings of Korea Institute of Construction Engineering and Management Annual Conference; 2010 Nov 5-6; Incheon, Korea. Seoul (Korea), Korea Institute of Construction Engineering and Management; c2010. p. 19-20.

6. Lee MK, Ahn CS, Kim YJ, Kim SA, Yoon SW, Chin SY. A Proposal on Efficient Construction Site Work Management Plan Using Smart-Phone. Proceedings of Korea Institute of Construction Engineering and Management Annual
Conference; 2010 Nov 5-6; Incheon (Korea). Seoul (Korea), Korea Institute of Construction Engineering and Management; c2010. p. 317-8.

7. Kim EJ, Park MS, Lee HS. Suggestion of Safety Management Smartphone Application based on PMIS. Proceedings of Architectural Institute of Korea Annual Conference; 2010 Oct 23; Cheongju (Korea). Seoul (Korea), Architectural Institute of Korea; c2010. p. 343-4.

8. Choi YG, Hwang KH, Lee MK, Kim SA, Yoon SW, Chin SY. The Plan Using an Auto Information Sensor for an Efficient Construction Information Gathering and Recording. Proceedings of Korea Institute of Construction Engineering and Management Annual Conference; 2010 Nov 5-6; Incheon, Korea. Seoul (Korea), Korea Institute of Construction Engineering and Management; c2010. p. 168-9.

9. Lee KP, Lee HS, Park MS, Kim EJ. Construction Material Management Using Smart Mobile Computing. Journal of Korea Institute of Construction Engineering and Management. 2011 June;12(2):59-69.

10. Kim YJ, Ahn CS, Choi YG, Kim SA, Yoon SW, Chin SY. A Study on Method to Progress Management based Augmented Reality(AR) on Smartphone. Proceedings of Korea Institute of Construction Engineering and Management Annual Conference; 2010 Nov 5-6; Incheon, Korea. Seoul (Korea), Korea Institute of Construction Engineering and Management; c2010. p. 156-7.

11. Chang JR, Hsu HM, Chao SJ. Development of a Road Monitoring and Reporting System Based on Location-Based Services and Augmented Reality Technologies. ASCE Journal of Performance of Constructed Facilities. Not printed (Accepted on August 1, 2011)

12. Lee WJ, Choi WW. A Case of Smart Phone Application to Construction Industry. Korean Journal of Institute of Construction Engineering and Management. 2010 Oct;11(5):30-4

13. Lee WJ, Choi WW. A Study of GS E\&C's Project Management System in Using of Smart Phone. Review of Architecture and Building Science. 2011 Jan;55(1):54-7. 